\title{
Investigations of the extended radio galaxy NGC 6251 and the galaxies in the environment
}

\author{
M. A. Hovhannisyan ${ }^{1 *}$, R. R. Andreasyan², G. M. Paronyan ${ }^{2}$ \\ ${ }^{1}$ NAS RA Institute of Applied Problems of Physics, Armenia \\ ${ }^{2}$ NAS RA V. Ambartsumian Byurakan Astrophysical Observatory (BAO), Armenia \\ *E-mail: martin@bao.sci.am
}

\begin{abstract}
The environment of one of the most extended radio galaxies, NGC 6251 , has been studied. The surface density of the environment galaxies in the region with the radius 300 arcmin around a radio galaxy NGC 6251 is 0.0315 in one $\operatorname{arcmin}^{2}$. In the distances less than 180 arcmin, the density of galaxies decreases, which is most likely due to the strong radio loudness and the interaction of radio galaxy with its surroundings.
\end{abstract}

Keywords: radio galaxies - NGC6251 - density of galaxies.

\section{Introduction}

There is a strong interaction between extended radio galaxies and galaxies in the neighborhood. In the vicinity of extended radio galaxies, there is a great number of objects that probably are connected together. The neighborhood of 35 investigated extended radio galaxies differs from the common field, as well as from each other. But the difference of behavior of each other mainly is not so strong. Around 4 objects from the 35 extended radio galaxies there is a deficiency of galaxies in a such large scale that we can think about the existence of large scale voids in the space. For the detailed study of these regions will be dedicated a separate work. In the vicinity of some other extended radio galaxies, there is great number of abundance of objects comparing with the field. The results of investigation of these regions are also very interesting, but it needs a study of another character.

\section{The environment of the radio galaxy NGC 6251}

This work is dedicated to the investigation of one of the most extended radio galaxies, NGC 6251. Around the extended radio galaxy NGC 6251, 
the density of neighboring galaxies is similar to majority of radio galaxies. 28 of the above-mentioned 35 extended radio galaxies exhibit similar behavior. NGC 6251 is the largest radio galaxy in the Northern sky. Its sizes reach up to 1.2 degrees (Waggett 1977). This radio galaxy is one of the most active ones in the region of all electromagnetic waves and has also large morphological varieties (Cohen 1977; Mingo 2014; Aswathy 2018; Croston 2018; Tseng 2016; Hovatta 2014; Babyk 2018).

We have investigated the environment objects of NGC 6251 (double galaxies, triples, groups, clusters, quasars), the density of these objects depending of the distances from the radio galaxy.

In the Table 1 there are galaxies and the densities of galaxies in the region of 300 arcmin around NGC 6251.

\begin{tabular}{|c|c|c|}
\hline Region & Galaxies & Density \\
\hline $90-120$ & 225 & 0.0114 \\
\hline $120-180$ & 613 & 0.0108 \\
\hline $180-240$ & 1303 & 0.0218 \\
\hline $240-300$ & 5981 & 0.0588 \\
\hline
\end{tabular}

Table 1: Galaxies and the densities of galaxies in the region of 300 arc.min. around NGC6251

The distance of NGC 6251 is about $100 \mathrm{Mpc}$ and its linear dimension is about $2 \mathrm{Mpc}$. We discus the existence of galaxies in the region 20-25 Mpc around the radio galaxy. As we have not all distances of galaxies in this region, so we use the angular distances for the environment galaxies. The galaxies of this region have a large influence on the radio galaxy, and the radio galaxy in turn has a strong influence on the galaxies around. This influence is large on the activity as well as on the morphology of galaxies. We suppose that in spite of the fact that these influences are significant, but most of the properties of galaxies are initial and exist from the beginning of formation of these objects.

The surface density of galaxies in the region with the radius 300 arcmin around the radio galaxy NGC 6251 is 0.0315 in one $\operatorname{arcmin}^{2}$. In the distances less than 180 arcmin, the density of galaxies decreases, which is most likely due to the large proportion of radio loudness and the interaction of radio galaxy with its surroundings.

\section{References}

Aswathy, S.; Ravikumar, C. D. 2018, MNRAS, 477, 2399

Babyk, Iu. V.; McNamara, B. R.; Nulsen, P. E. J.; Hogan, M. T.; 
Vantyghem, A. N.; Russell, H. R.; Pulido, F. A.; Edge, A. et al. 2018, ApJ., 857, 32

Cohen, M.; Readhead, A. 1977, ApJ, 233, L101

Croston, J. H.; Ineson, J.; Hardcastle, M. J. 2018, MNRAS, 476, 1614

Hovatta, T.; Pavlidou, V.; King, O. G.; Mahabal, A.; Sesar, B.;

Dancikova,R.; Djorgovski, S. G.; Drake, A.; Laher, R.; Levitan, D.; Max-Moerbeck,W.; Ofek, E. O.; Pearson, T. J.; Prince, T. A.; Readhead, A. C. S.; Richards, J. L.; Surace, J. 2014, MNRAS 439, 690

Mingo, B.; Hardcastle, M. J.; Croston J.H.Dicken D.; Evans D. A.,Morganti R.,Tadhunter C. A 2014, MNRAS, 440, 269

Tseng, C.-Y.; Asada, K.i; Nakamura, M.; Pu, H.-Yi; Algaba, J.-C.; Lo, W.-P. 2016, ApJ, 833, 288

Waggett, P. C., Warner, P. J., Baldwin, J. E. 1977, MNRAS, 181, 465 